\title{
Maximum a Posteriori vs Maximum Probability Recursive Sparse Estimation
}

\author{
Lachlan Blackhall and Michael Rotkowitz
}

\begin{abstract}
Recursive sparse parameter estimates obtained using the author's recent maximum a posteriori (MAP) approach, where the sparse parameter estimates are determined as the a posteriori mode of a Gaussian sum filter, are compared with a new maximum probability (MP) methodology, where the sparse parameter estimates are determined as the component of a Gaussian sum filter with the highest a posteriori weighting. We show how the performance of the MP estimator approach to sparse parameter estimates, in both sparsity and mean square error senses, depends on the parameters that characterize each multivariate Gaussian in the Gaussian sum filter. Through this work we also provide additional performance analysis for the MP estimator and suggest possible areas of future work that will further improve its performance.
\end{abstract}

\section{INTRODUCTION}

As regression and estimation problems become more complex it is common to encounter parameter estimation problems with a large number of candidate parameters being equal to zero. Estimation problems of this form, where the number of parameters $q$ we are considering is greater than the number necessary to explain the data are said to be sparse. In these situations it is desirable to use an estimator that will systematically produce sparse estimates. This is of practicable interest as a high degree of sparsity corresponds to simpler models. The best way to summarize the myriad reasons why sparse estimates are often desirable, is that there are typically costs associated with the cardinality of the parameter which are not explicitly stated in the objective.

The design and implementation of a recursive sparse estimator has recently been presented by the authors [1] and emerged from the results of Candès, Tao and Romberg (a good survey of the results is given in [2] and [3]). This work has provided significant insight into a methodology which has been widely known as the LASSO ([4]) in the statistical domain. This can now be considered as a special case of what is often referred to as ' $\ell_{1}$-Magic'; that is, the tendency of $\ell_{1}$ minimization or penalization to produce parsimonious results in problems where enforcing that directly would yield computational intractability. The conditions and reasons for which this occur have become much better understood in recent years (see the references cited above).

The authors previous work [1] set out the framework for an estimation algorithm that was able to return sparse parameter estimates recursively using a maximum a posteriori (MAP)

L. Blackhall is with The Research School of Information Sciences and Engineering, The Australian National University, Canberra, ACT, 0200, Australia lachlan.blackhalleanu.edu.au

M. Rotkowitz is with The Department of Electrical and Electronic Engineering, The University of Melbourne, Parkville, VIC, 3010, Australia mcrotkeunimelb.edu.au approach. In this approach the sparse parameter estimates are determined as the a posteriori mode of a Gaussian sum filter. This framework required a threshold to obtain the sparse parameter estimates. In this work we extend the previous result and show how it is possible to achieve exactly sparse parameter estimates, that is parameter estimates that are exactly equal to zero. In achieving this we present a maximum probability (MP) recursive sparse estimator, where the sparse parameter estimates are determined as the component of a Gaussian sum filter with the highest a posteriori weighting. Through this analysis we are able to make clear the connection between the attainable sparsity and the filter variables using the new maximum probability methodology. We will begin with some preliminaries as well as outlining the original recursive sparse estimator of [1] before describing the MP estimator and providing numerical examples comparing the MAP and MP estimators.

\section{PRELIMINARIES}

\section{A. Distributions}

We will make extensive use of the multivariate Gaussian or normal distribution throughout this paper and we give the following standard definition.

Definition 1: Given a mean $\mu \in \mathbb{R}^{n}$ and a covariance $B \in \mathbb{R}^{n \times n}$ with $B>0$, we say that a random variable $X$ is normally distributed and denote $X \sim \mathcal{N}(\mu, B)$ if it has the following probability density function (pdf) for all $x \in \mathbb{R}^{n}$ (where $x$ is a realization of $X$ )

$\mathcal{N}(x ; \mu, B)=\frac{1}{(2 \pi)^{N / 2}|B|^{1 / 2}} \exp \left(-\frac{1}{2}\left\|B^{-1 / 2}(x-\mu)\right\|_{2}^{2}\right)$

where $|B|=\operatorname{det}(B)$.

We also introduce the Laplace, or double exponential, distribution.

Definition 2: Given a mean $\mu \in \mathbb{R}$ and a scale parameter $\tau>0$, we say that a random variable $X$ has the Laplace or double exponential distribution and denote $X \sim \mathcal{L}(\mu, \tau)$ if it has the following pdf for all $x \in \mathbb{R}$

$$
\mathcal{L}(x ; \mu, \tau)=\frac{1}{2 \tau} \exp \left(-\frac{|x-\mu|}{\tau}\right)
$$

In this paper we only consider Laplace distributions with zero mean, and thus abbreviate our notation as $\mathcal{L}(\tau) \sim \mathcal{L}(0, \tau)$ and $\mathcal{L}(x ; \tau)=\mathcal{L}(x ; 0, \tau)$.

\section{B. Regression}

We consider the following parameter estimation problem. Given $X \in \mathbb{R}^{N \times q}$, the rows of which are independent 
explanatory variables, and dependent response variables, or observations $y \in \mathbb{R}^{N}$, we assume that the observations are generated as

$$
y=X \theta+\varepsilon,
$$

where the noise may be considered normally distributed as $\varepsilon \sim \mathcal{N}(0, R)$, for some $R \in \mathbb{R}^{N \times N}, \quad R>0$. Typically the noise will be considered independent, and we then have $R=\sigma_{\varepsilon}^{2} I$ for some $\sigma_{\varepsilon}>0$. We then seek to estimate the underlying parameters $\theta \in \mathbb{R}^{q}$.

An overview of the solutions to this standard regression problem can be found in [1], thus only a short overview is included herein. Typical solutions for the regression problem have utilized the (ordinary) least squares (OLS) estimator. Since the least squares estimator only considers the goodness-of-fit, it tends to overfit the data. Shrinking the parameter, such as, by penalizing its size, typically performs better on out-of-sample data. A general way to achieve this is to enforce such a penalty as

$$
\theta^{*}=\arg \min _{\hat{\theta}}\|y-\hat{y}\|_{2}^{2}+\lambda\|\hat{\theta}\|_{p}^{p}
$$

for some parameter $\lambda \geq 0$ and some norm $p \geq 1$, where $\hat{y}=$ $X \hat{\theta}$ gives the fitted values. When we consider this estimator with $p=2$, it becomes known as ridge regression $(\boldsymbol{R R})$, regularized least squares or Tikhonov regularization ([5]). Both the OLS and RR estimators have a known closed form solution [1].

If we solve (4) for $p=1$, that estimator is known as the LASSO ([4]). While a closed-form solution does not exist in general, solving for $\theta^{*}$ is still a convex optimization problem and readily solved. This estimator has a Bayesian interpretation; namely, that it arises as the maximum a posteriori (MAP) estimate if the parameters have independent prior distributions of $\theta_{i} \sim \mathcal{L}\left(2 \sigma_{\varepsilon}^{2} / \lambda\right)$. It is this estimator that was used as the basis for the recursive sparse estimator developed in [1].

\section{Recursive Estimation And the Gaussian Sum FILTER}

\section{A. Recursive Parameter Estimation}

If the number of observations $N$ is very large or on-line estimates are needed as the data becomes available it is often desirable to recursively obtain the parameter estimates. A more detailed outline of recursive estimation can be found in [1].

The LASSO, however, has no such recursive estimator, as a double exponential prior distribution yields a posterior which is not a double exponential nor any other easily characterizable distribution. The same is true for the other priors ([6]) known to induce sparse MAP estimates. It was shown in [1] how it is possible to achieve recursive sparse estimation and an outline of this development is presented in the following sections.

\section{B. The Gaussian Sum Filter}

We outlined in [1] that a parameter having a prior distribution that is Gaussian is easily recursively estimated, as the distribution can be simply parameterized as a mean and covariance. The Kalman filter is the most well known recursive estimator in this instance. This same simple parametrization extends to multivariate Gaussian distributions in many dimensions. An extension of the Kalman filter is the Gaussian sum filter that allows non-Gaussian filtering to leverage the effectiveness of the Kalman filter. The Gaussian sum filter was outlined in [7] and [8] and further detailed in [9]. We will outline the form of the filter below and our development is based upon a more general version in [9].

Using the Gaussian sum filter for parameter estimation or system identification can be considered a special case of the filter as it is typically used for state estimation. This is achieved by assuming that there are no internal system dynamics and thus the parameter estimates can only change when a new measurement is obtained. The Gaussian sum filter can be considered as a weighted bank of Kalman filters operating in parallel, where the weights change after each measurement is processed.

We assume a linear measurement model:

$$
y_{k}=X_{k} \theta+\varepsilon_{k}
$$

where we have a Gaussian measurement noise process $\left(\varepsilon_{k} \sim\right.$ $\left.\mathcal{N}\left(0, R_{k}\right)\right)$ and a prior distribution of $\theta$ given by:

$$
\theta \sim \sum_{i=1}^{M} \alpha_{i} \mathcal{N}\left(\mu_{i}, B_{i}\right)
$$

where $\mu_{i}$ and $B_{i}$ are the $q$-dimensional mean vector and $q \times q$ covariance matrix respectively.

Let us now assume that at a given point we receive a new measurement $y_{k}$, along with its corresponding explanatory variable $X_{k}$, and that the distribution of the parameter given all of the previous measurements is given as:

$$
\theta \mid Y_{k-1} \sim \sum_{i=1}^{M} \alpha_{i, k-1} \mathcal{N}\left(\mu_{i, k-1}, B_{i, k-1}\right)
$$

where $Y_{k}=\left[y_{1}, \ldots, y_{k}\right]$ again represents all of the measurements up to and including $k$. The distribution of the parameter given all of the measurements including the new one is then given by:

$$
\theta \mid Y_{k} \sim \sum_{i=1}^{M} \alpha_{i, k} \mathcal{N}\left(\mu_{i, k}, B_{i, k}\right)
$$

where the updated weights $\alpha_{i, k}$, means $\mu_{i, k}$, and covari- 
ances $B_{i, k}$ are given by:

$$
\begin{aligned}
\Omega_{i, k} & =X_{k}^{T} B_{i, k-1} X_{k}+R_{k} \\
K_{i, k} & =B_{i, k-1} X_{k} \Omega_{i, k}^{-1} \\
B_{i, k} & =B_{i, k-1}-B_{i, k-1} X_{k} \Omega_{i k}^{-1} X_{k}^{T} B_{i, k-1}^{T} \\
\hat{y}_{i, k} & =X_{k} \mu_{i, k-1} \\
\mu_{i, k} & =\mu_{i, k-1}+K_{i, k}\left(y_{k}-\hat{y}_{i, k}\right) \\
\alpha_{i, k} & =\frac{\alpha_{i, k-1} \mathcal{N}\left(y_{k} ; \hat{y}_{i, k}, \Omega_{i, k}\right)}{\sum_{j=1}^{M} \alpha_{j, k-1} \mathcal{N}\left(y_{k} ; \hat{y}_{j, k}, \Omega_{j, k}\right)}
\end{aligned}
$$

If we have a Gaussian mixture for our prior distribution given as (6), we can then set the initial weights as $\alpha_{i, 0}=\alpha_{i}$, the initial means as $\mu_{i, 0}=\mu_{i}$, and the initial covariances as $B_{i, 0}=B_{i}$ for all $i \in\{1, \ldots, M\}$, run the above iteration for each new measurement received, and then arrive at the posterior distribution as

$$
\theta \mid Y_{N} \sim \sum_{i=1}^{M} \alpha_{i, N} \mathcal{N}\left(\mu_{i, N}, B_{i, N}\right) .
$$

Finding the MAP estimate of $\theta$ then requires finding the mode of this posterior Gaussian mixture which is given by:

$$
\theta_{\mathrm{MAP}}^{*}=\arg \max _{\hat{\theta}} \sum_{i=1}^{M} \alpha_{i, N} \mathcal{N}\left(\hat{\theta} ; \mu_{i, N}, B_{i, N}\right) .
$$

\section{Gaussian MiXtures AND the Laplacian Prior}

As mentioned in the preliminaries, the LASSO estimate can be interpreted as the MAP estimate when the parameters have independent Laplace prior distributions. It was shown in [1] that the higher dimensional Laplacian prior could be approximated as a Gaussian sum by:

$$
\begin{aligned}
f_{0}(\theta) & =\prod_{j=1}^{q} \mathcal{L}\left(\theta_{j} ; \tau\right) \\
& =\prod_{j=1}^{q} \int_{\psi_{j}=0}^{\infty} g\left(\psi_{j} ; \tau\right) \mathcal{N}\left(\theta_{j} ; 0, \psi_{j}\right) d \psi_{j} \\
& \approx \prod_{j=1}^{q} \sum_{i_{j}=1}^{M} \alpha_{i_{j}} \mathcal{N}\left(\theta_{j} ; 0, \psi_{i_{j}}\right) \\
& =\sum_{i_{1}=1}^{M} \cdots \sum_{i_{q}=1}^{M}\left(\prod_{j=1}^{q} \alpha_{i_{j}} \mathcal{N}\left(\theta_{j} ; 0, \psi_{i_{j}}\right)\right) \\
& =\sum_{i_{1}=1}^{M} \cdots \sum_{i_{q}=1}^{M}\left(\prod_{j=1}^{q} \alpha_{i_{j}} \prod_{j=1}^{q} \mathcal{N}\left(\theta_{j} ; 0, \psi_{i_{j}}\right)\right) \\
& =\sum_{i_{1}=1}^{M} \cdots \sum_{i_{q}=1}^{M} \alpha_{i_{1}, \ldots, i_{q}} \mathcal{N}\left(\theta ; 0, B_{i_{1}, \ldots, i_{q}}\right)
\end{aligned}
$$

where $g\left(\psi_{j} ; \tau\right)$ is the exponential hyper prior and the multivariate weightings are given as

$$
\alpha_{i_{1}, \ldots, i_{q}}=\prod_{j=1}^{q} \alpha_{i_{j}}
$$

and the multivariate covariances as

$$
B_{i_{1}, \ldots, i_{q}}=\operatorname{diag}\left(\psi_{i_{1}}, \ldots, \psi_{i_{q}}\right) .
$$

This shows how to approximate the prior distribution for the LASSO as a sum of Gaussian distributions. Thus we can utilize the Gaussian sum filter of Section III-B to recursively estimate the parameters with each new observation, which is not possible with the original distribution.

We unfortunately see that if we have $q$ parameters to estimate and approximate each univariate double exponential with $M$ Gaussians, then we end up using $M^{q}$ total Gaussians in the final mixture. It was shown in [1] that we can set $M=2$ and obtain comparable results.

\section{The Bi-Gaussian Filter}

In [1] we saw that it was possible to set $M=2$ in the recursive sparse estimator and obtain equivalent, although marginally higher, levels of sparsity compared to the case when $M>>2$. In comparing the bi-Gaussian filter to the exposition seen in Eqs. 12-14 it can be seen that we now have two Gaussian distributions, chracterised by the variances $\sigma_{\min }^{2}$ and $\sigma_{\max }^{2}$ respectively and we typically use an a priori weighting of $\alpha=0.5$ for both Gaussians rather than the weighting given by the hyper prior $g\left(\psi_{j} ; \tau\right)$ as was done previously. This is a heuristic adjustment to the algorithm that represents the two potential outcomes for each parameter, that it is either zero or non-zero. If the parameter is nonzero we are interested in obtaining further Bayesian credible statistics. Conversely, if the parameter is zero then we need not consider it in the model. In our earlier work this left us needing to choose variances $\sigma_{\min }^{2}$ and $\sigma_{\max }^{2}$ as the variables that affected the performance of the recursive sparse filter. It should be observed that $\sigma_{\text {min }}^{2} \neq 0$ means that we are not representing the potential for a zero parameter estimate exactly but rather that the parameter is small but finite. It was possible to show that this assumption still allowed sparse estimates to be recovered [1]. Furthermore, it was necessary to set $\sigma_{\min }^{2} \neq 0$ in order to be able to compute a meaningful MAP estimate. We will show in Section V-A.1 that such a MAP estimate is no longer meaningful, from the perspective of parameter estimation, if $\sigma_{\min }^{2}=0$.

It is of interest to understand the conditions under which it is possible to still achieve meaningful sparse estimates when we set $\sigma_{\min }^{2}=0$ and this is the topic of subsequent sections.

\section{A. The zero-mean, zero-variance Impulse}

We are now interested in examining the effect, on the Gaussian sum filter, of setting $\sigma_{\min }^{2}=0$ to represent the possibility of the parameter estimate being exactly equal to zero. This introduces some further challenges which will be addressed below. The equations for the Gaussian sum filter are presented in Eq. 9 and we proceed by evaluating the effect on each line of the algorithm when we have a zeromean, zero variance impulse as one of the components of the $i$-th multivariate Gaussian.

For a given matrix $(B)$ we will use standard MATLAB notation to indicate elements within the matrix. Thus $B(j, l)$ 
is the $(j, l)$-th entry of the matrix $B$ and $B(j,:)$ and $B(:, j)$ represent the $j$-th row and column, respectively. Initially we can see that a zero-mean, zero-variance impulse as the $j$ th component of the $i$-th multivariate Gaussian implies that $\mu_{i}(j)=0, B_{i}(j,:)=0$, and $B_{i}(:, j)=0$.

Beginning with the definition of $\Omega_{i, k}=X_{k}^{T} B_{i, k-1} X_{k}+$ $R_{k}$ it is observed that the zero impulse component only influences the magnitude of $\Omega_{i, k}$ which it does through the first term of the previous equation.

The second line of the algorithm requires the computation of the vector $K$ and it is here that we first observe the effect of the impulse. It is clear from the form of the vector $K_{i, k}=B_{i, k-1} X_{k} \Omega_{i, k}^{-1}$ that:

$$
B_{i, k-1}(j,:)=0 \Rightarrow K_{i, k}(j,:)=0
$$

We now proceed to update the covariance matrix and we have the following form for the update $B_{i, k}=B_{i, k-1}-$ $B_{i, k-1} X_{k} \Omega_{i k}^{-1} X_{k}^{T} B_{i, k-1}^{T}$. If we have $B_{i, k-1}(j,:)=0$ and $B_{i, k-1}(:, j)=0$, that is the $j$-th row and column are already zero then we can see that:

$$
B_{i, k-1}(j,:)=0 \Rightarrow B_{i, k}(j,:)=0
$$

and

$$
B_{i, k-1}(:, j)=0 \Rightarrow B_{i, k}(:, j)=0
$$

highlighting that once the covariance matrix has a row and column initialized to zero, as in the case of the impulse, it remains that way for all time.

The penultimate step in the algorithm is to update the Gaussian mean and from $\mu_{i, k}=\mu_{i, k-1}+K_{i, k}\left(y_{k}-\hat{y}_{i, k}\right)$ it can be seen that $K_{i, k}(j,:)=0$ and $\mu_{i, k-1}(j)=0$ imply $\mu_{i, k}(j)=0$. Essentially we can see that a zero-mean, zerovariance impulse remains as such for all time. This makes intuitive sense as zero-variance implies zero uncertainty, and hence any change in the mean or variance would contradict this assertion.

The final step in the algorithm is the evaluation of the weighting term $\left(\alpha_{i, k}\right)$ for each multivariate Gaussian in the Gaussian sum filter. Essentially, in updating $\alpha_{i, k}$ we are determining how closely the mean $\left(\mu_{i, k}\right)$ of the $i$-th Gaussian explains the observation $\left(y_{k}\right)$. Thus larger values of $\alpha_{i, k}$ imply that the mean of the $i$-th Gaussian is a better estimate of the parameter vector of interest.

The analysis presented in this section shows that the Gaussian sum filter continues to provide meaningful output when a zero-mean, zero-variance component is used. It remains to show how to evaluate the multivariate Gaussian with a zero-mean, zero-variance component and this is the topic of the following section.

1) Evaluating a Gaussian Sum with a zero-mean, zerovariance Impulse: We saw earlier in Eq. 1 the form of the multivariate Gaussian distribution. For clarity it is repeated here:

$\mathcal{N}(x ; \mu, B)=\frac{1}{(2 \pi)^{N / 2}|B|^{1 / 2}} \exp \left(-\frac{1}{2}\left\|B^{-1 / 2}(x-\mu)\right\|_{2}^{2}\right)$
We first present two standard lemmas, without proof, about the computation of matrix determinants:

Lemma 3: Any matrix with a row or column equal to zero is singular.

Lemma 4: The determinant of a singular matrix is zero.

In our previous work [1] we were able to compute the parameter estimates by finding the MAP estimate directly from the resulting Gaussian sum using what the authors of [10] called the gradient-quadratic method.

Using the previous two lemmas it is clear that when a zero-mean, zero-variance impulse is used as a component in the Gaussian sum filter the term $|B|^{-1 / 2}$ goes to infinity, implying that obtaining a MAP estimate from this Gaussian sum is now not useful in determining an accurate parameter estimate.

An alternative approach, similar to that in [11], that is suitable for a Gaussian sum with zero-mean, zero-variance components, is determining the component of the gaussian mixture with the largest weighting. We will refer to this as the maximum probability (MP) estimate. In this methodology we can interpret $\alpha_{i, k}$ as the measure of accuracy of the mean $\left(\mu_{i, k}\right)$ of the $i$-th multivariate Gaussian being the best estimate of the true parameter values $\theta$. From this perspective it is possible to compute the sparse estimate as the mean of the multivariate Gaussian that has the largest weighting $\alpha_{i, k}$ and thus our maximum probability estimator is given by:

$$
\begin{aligned}
i^{*} & =\arg \max _{i} \alpha_{i, N} \\
\theta_{\mathrm{MP}}^{*} & =\mu_{i^{*}, N}
\end{aligned}
$$

This can be compared to the earlier MAP estimate given in Eq. 11. It is this maximum probability methodology that we will use to compare with the earlier recursive sparse estimator implementation of [1].

The maximum probability approach has an alternate interpretation as the estimator where we perform $2^{q}$ regressions and choose at each time step the regression output that maximizes some performance measure. There are some other points of interest in comparing between the two filters. In the original filter it was necessary to determine at each time step the mode of the Gaussian sum in order to determine the parameter estimates. This was a computationally expensive step as even with the approach detailed in [10] we are forced to compute gradient or fixed point search in a high dimensional space. In moving to the maximum probability estimate we are no longer required to determine the mode resulting in a computationally simpler task. In the following section we present numerical comparisons of the two estimator implementations in order to understand the effect on performance of moving from maximum a posteriori (MAP) to maximum probability (MP) estimate.

\section{SPARSITY AND PERformance ANALYSis}

\section{A. Maximum a Posteriori (MAP) vs Maximum Probability (MP) Estimates}

We now compare the original recursive sparse estimator [1] with the maximum probability estimator (for both $\sigma_{\min }^{2}=$ 
0 and $\sigma_{\min }^{2} \neq 0$, where we choose $\sigma_{\min }^{2}=1 \times 10^{-4}$ ) as well as the least squares estimator (OLS), ridge regression (RR), and of course, the LASSO. In these examples we have $M=2, q=10$ and we simulated fifty data sets where we have $N=30$ data points. The probability of each parameter being equal to zero was 0.5 . The non-zero parameters were then chosen from a uniform distribution on the interval $[0,5]$. The regressor matrix is composed of random values drawn from the uniform distribution on the unit interval (that is, $X_{i j} \sim U[0,1]$, generated using the MATLAB rand command), the measurement noise is generated (using the MATLAB randn command) as $\varepsilon_{k} \sim$ $\mathcal{N}\left(0, \sigma_{\varepsilon}^{2}\right)$ with $\sigma_{\varepsilon}^{2}=0.5$, and the measurements are then generated as $y_{k}=X_{k} \theta+\varepsilon_{k}$. Fixed random seeds were chosen such that the results shown in Table I are directly comparable. In all further results these random seeds are maintained to allow direct comparison of the various approaches outlined in this paper.

We now outline the implementation of the recursive sparse estimator detailed in this paper. The prior of each parameter is assumed to be independently distributed and represented as a sum of two Gaussian distributions with $\sigma_{\min }^{2}$ as outlined above, $\sigma_{\max }^{2}=25$ and equal apriori weightings $(\alpha=0.5)$ for both variance components. The mean of each multivariate Gaussian is a $1 \times q$ vector of zeros.

As presented previously, $\lambda$ is the penalty term for the one norm of the parameters in the LASSO. We choose to implement the recursive sparse estimator with equal a priori weightings ( $\alpha=0.5)$ and thus we are only required to choose the penalty terms $(\lambda)$ for the LASSO and the ridge regression. The penalty terms were chosen by two-fold cross validation for the case where five of the parameters were equal to zero. Utilizing this method we obtain $\lambda=0.5$ as the penalty parameter for the LASSO and we similarly obtain $\lambda=0.05$ as the penalty term for the ridge regression.

\begin{tabular}{|c|c|c|c|}
\hline Method & $\begin{array}{c}\text { Median } \\
\text { MSE }\end{array}$ & $\begin{array}{c}\text { Perc. True } \\
\text { Zero Coeffs. }\end{array}$ & $\begin{array}{c}\text { Perc. False } \\
\text { Zero Coeffs. }\end{array}$ \\
\hline OLS & 0.075 & $0 \%$ & $0 \%$ \\
RR & 0.08 & $0 \%$ & $0 \%$ \\
LASSO & 0.046 & $12 \%$ & $3 \%$ \\
RS(MAP) & 0.049 & $94 \%$ & $26 \%$ \\
RS(MP) & 0.030 & $95 \%$ & $14 \%$ \\
RS(MP, $\left.\sigma_{\min }^{2}=0\right)$ & 0.030 & $95 \%$ & $14 \%$ \\
\hline
\end{tabular}

TABLE I: A comparison of recursive sparse estimators using both MAP and MP methods of estimating the sparse estimate when $q=10$. Results for the MP criterion are shown for both $\sigma_{\text {min }}^{2}=0$ and $\sigma_{\text {min }}^{2} \neq 0$. The estimates obtained using these methods are further compared to the ordinary least squares (OLS), Ridge regression (RR) and the LASSO.

The LASSO is implemented using code from [12], and computation of the least squares and ridge regression estimators is straightforward. The comparison of the results from using this algorithm with different parameter combinations can be seen in Table I. For each choice of parameters and regression algorithm we have computed the median mean squared error (MSE) of the coefficient estimates, percentage of actual zero parameters correctly estimated to be zero (Perc. True Zero Coeffs.) and the percentage of actual nonzero parameters incorrectly estimated to be zero (Perc. False Zero Coeffs.).

In Table I we observe that the MP implementation of the recursive sparse filter achieves a high level of accuracy in its own right as well as comparing favourably with the other estimators, including the MAP version of the same filter. For both $\sigma_{\min }^{2} \neq 0$ and $\sigma_{\min }^{2}=0$ the maximum probability estimate is identical. This arises because whether starting with $\sigma_{\text {min }}^{2}=0$ or a value very close to zero, $\sigma_{\min }^{2} \neq 0$, this component of the Gaussian mixture will have the highest weighting and will thus be chosen when using the MP methodology for estimation.

Of most interest is the decrease in the percentage of false coefficients when moving from the MAP to MP methodologies. Previously, when using the MAP methodology, the Gaussian components with $\sigma_{\min }^{2}$ placed a large density around the zero estimate, resulting in sparse parameter estimates being favored. Conversely when we use the MP approach we are no longer relying on a mode estimate and thus we are choosing the estimator that best fits the measurements. This reduces the dependence of the estimator output on the value of $\sigma_{\min }^{2}$, making it more likely to accurately choose the estimate with the correct level of sparsity and resulting in the observed decrease in false zero coefficients.

Given the results of this comparison it is of interest to better understand how the initial choice of $\alpha_{0}$, where $\alpha_{0}$ is the prior probability given to the sparsity inducing zeromean, zero-variance $\left(\sigma_{\min }^{2}=0\right)$ impulse and $\sigma_{\max }^{2}$ affect the resultant sparsity when we use the maximum probability method of achieving sparse estimates. This analysis is the subject of the following section.

\section{B. The effect of initial choices of $\alpha_{0}$ and $\sigma_{\max }^{2}$ on the resultant sparsity.}

We now extend the analysis of the MP method of achieving sparse estimates by analyzing the effect on the resultant sparsity as $\alpha_{0}$ and $\sigma_{\max }$ are varied. We use the same approach as before and the analysis is identically carried out, except that now we vary, independently, first $\alpha_{0}$ then $\sigma_{\text {max }}^{2}$. We present results for sparsity levels when we only change $\sigma_{\max }^{2}$ (Table II), only change initial weight $\alpha$ (Table III) and then change both $\sigma_{\max }^{2}$ and $\alpha_{0}$ (Fig. 1).

As $\sigma_{\max }^{2}$ increases we achieve increased sparsity. When $\sigma_{\max }^{2}$ is closer to zero there is a higher probability that the Gaussian component corresponding to a non-zero parameter estimate will best explain the measurements of a actual zero parameter, resulting in non-zero and hence, nonsparse parameter estimates. Consequently, as $\sigma_{\max }^{2}$ increases this likelihood decreases. Additionally as $\sigma_{\max }^{2}$ increases we are essentially increasing the uncertainty associated with the corresponding multivariate Gaussian. This penalizes the corresponding weighting and is thus applied most heavily to less sparse multivariate Gaussians (estimates with more Gaussian components with variance $\sigma_{\max }^{2}$ ), resulting in the increased sparsity observed. 


\begin{tabular}{|c|c|c|c|}
\hline$\sigma_{\max }^{2}$ & $\begin{array}{c}\text { Median } \\
\text { MSE }\end{array}$ & $\begin{array}{c}\text { Perc. True } \\
\text { Zero Coeffs. }\end{array}$ & $\begin{array}{c}\text { Perc. False } \\
\text { Zero Coeffs. }\end{array}$ \\
\hline 1 & 0.067 & $61 \%$ & $6 \%$ \\
2.2 & 0.052 & $78 \%$ & $11 \%$ \\
4.6 & 0.037 & $85 \%$ & $14 \%$ \\
10 & 0.041 & $89 \%$ & $21 \%$ \\
21.5 & 0.018 & $94 \%$ & $19 \%$ \\
46.4 & 0.025 & $95 \%$ & $21 \%$ \\
100 & 0.030 & $95 \%$ & $18 \%$ \\
215.4 & 0.028 & $98 \%$ & $25 \%$ \\
464.2 & 0.031 & $98 \%$ & $21 \%$ \\
1000 & 0.042 & $97 \%$ & $29 \%$ \\
\hline
\end{tabular}

TABLE II: A comparison of the performance of the recursive sparse estimator using the MP method of obtaining the sparse estimates when $q=10, \alpha_{0}=0.5, \sigma_{\min }^{2}=0$ and $\sigma_{\max }^{2}$ is varied logarithmically between $\sigma_{\max }^{2}=1$ to $\sigma_{\max }^{2}=1000$.

Similarly when we increase the initial weighting on the sparsity inducing impulse we find a similar increase in resultant sparsity. In this instance we are now directly biasing the impulse to increase the likelihood of achieving sparse estimates and this is exactly what is achieved in the results shown in Table III.

\begin{tabular}{|c|c|c|c|}
\hline$\alpha_{0}$ & $\begin{array}{c}\text { Median } \\
\text { MSE }\end{array}$ & $\begin{array}{c}\text { Perc. True } \\
\text { Zero Coeffs. }\end{array}$ & $\begin{array}{c}\text { Perc. False } \\
\text { Zero Coeffs. }\end{array}$ \\
\hline 0.05 & 0.078 & $0 \%$ & $0 \%$ \\
0.15 & 0.078 & $51 \%$ & $10 \%$ \\
0.25 & 0.044 & $80 \%$ & $12 \%$ \\
0.35 & 0.048 & $84 \%$ & $21 \%$ \\
0.45 & 0.020 & $93 \%$ & $18 \%$ \\
0.55 & 0.024 & $95 \%$ & $20 \%$ \\
0.65 & 0.029 & $95 \%$ & $18 \%$ \\
0.75 & 0.028 & $98 \%$ & $25 \%$ \\
0.85 & 0.031 & $98 \%$ & $21 \%$ \\
0.95 & 0.042 & $99 \%$ & $31 \%$ \\
\hline
\end{tabular}

TABLE III: A comparison of the performance of the recursive sparse estimator using the MP method of obtaining the sparse estimates when $q=10, \sigma_{\max }^{2}=25, \sigma_{\min }^{2}=0$ and $\alpha_{0}$ is varied linearly between $\alpha_{0}=0.05$ to $\alpha_{0}=0.95$. $\alpha_{0}$ is the prior probability given to the sparsity inducing zero-mean, zero-variance $\left(\sigma_{\min }^{2}=0\right)$ impulse.

What is of interest in both sets of results is that where we increase $\sigma_{\max }^{2}$ or $\alpha_{0}$ above a certain level the percentage of true zero coefficients only increases slowly whereas the percentage of false zero coefficients increases more quickly. In Fig. 1 both $\sigma_{\max }^{2}$ and $\alpha_{0}$ are varied simultaneously and again we can see that there is a region where the increase in both $\sigma_{\max }^{2}$ and $\alpha_{0}$ increases the percentage of false coefficients much more quickly than the percentage of true coefficients. This suggests that it is possible to choose the $\sigma_{\max }^{2}$ and $\alpha_{0}$ that correspond to the level of acceptable true and false zero parameter estimates for a given sparse estimation problem.

\section{CONCLUSION AND FUTURE WORK}

This paper compares the maximum a posteriori approach to recursive sparse estimation with a maximum probability approach that recursively provides exactly sparse estimates. The results presented show that the MP estimation methodology compares favourably with the existing MAP

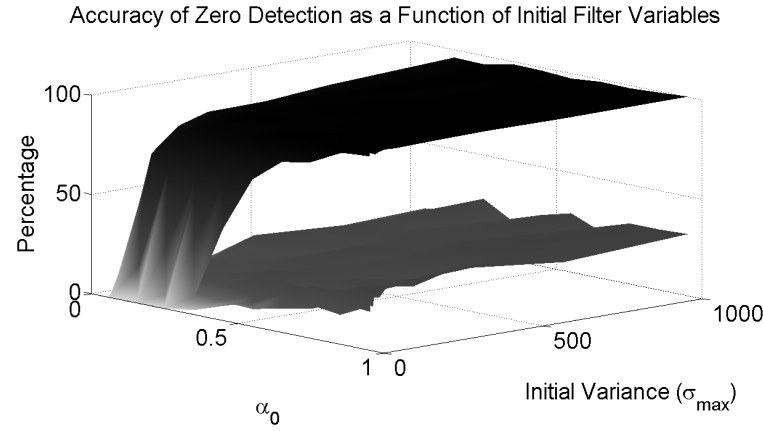

Fig. 1: Comparison of correctly determined zeros (upper surface) and incorrectly determined zeros (lower surface) as the initial $\sigma_{\max }^{2}$ and $\alpha_{0}$ are varied.

methodology while providing some important computational efficiency.

The effect on the resultant sparsity of varying the filter variables in the maximum probability approach has been presented, providing an initial characterization of the attainable sparsity performance of the MP estimator. The analysis suggests that for a given level of desired sparsity there is an appropriate combination of $\alpha_{0}$ and $\sigma_{\max }^{2}$ that minimizes incorrectly estimated zero coefficients.

Future work in this area will be to optimize the performance of the filter to allow sparse estimation for very large parameter estimation problems as well as applying this sparse estimation methodology to real data sets.

\section{REFERENCES}

[1] L. Blackhall and M. Rotkowitz, "Recursive sparse estimation using a gaussian sum filter," in Proceedings of the International Federation for Automatic Control Congress, 2008.

[2] E. J. Candès, "Compressive sampling," in Proceedings of the International Congress of Mathematicians. European Mathematical Society, 2006.

[3] D. L. Donoho and J. Tanner, "Sparse nonnegative solution of underdetermined linear equations by linear programming," Technical Report, 2005.

[4] R. Tibshirani, "Regression shrinkage and selection via the lasso," Journal of the Royal Statistical Society: Series B, vol. 58, no. 1, pp. 267-288, 1996.

[5] A. N. Tikhonov, "Solution of incorrectly formulated problems and the regularization method," Sov. Math., Dokl., vol. 5, pp. 1035-1038, 1963.

[6] J. Griffin and P. Brown, "Alternative prior distributions for variable selection with very many more variables than observations," University of Kent Technical Report, 2005.

[7] H. Sorenson and D. Alspach, "Recursive Bayesian estimation using Gaussian sums," Automatica, vol. 7, pp. 465-479, 1971.

[8] D. L. Alspach and H. W. Sorenson, "Nonlinear Bayesian estimation using Gaussian sum approximations," IEEE Transactions on Automatic Control, vol. AC-17, no. 4, pp. 439-448, August 1972.

[9] B. D. Anderson and J. B. Moore, Optimal Filtering. Dover Publications, Inc, 2005

[10] M. A. Carreira-Perpinán, "Mode-finding for mixtures of Gaussian distributions," IEEE Transactions on Pattern Analysis and Machine Intelligence, vol. 22, no. 11, pp. 1318-1323, November 2000.

[11] P. Stoica and Y. Selen, "Model-order selection: A review of information criterion rules," IEEE Signal Processing Magazine, vol. 21, no. 4, pp. 36-47, July 2004.

[12] M. Schmidt, "Lasso matlab implementation (http://www.cs.ubc.ca/ schmidtm/software/lasso.html)," 2005. 